Die kürzesten Worte, nämlich JA und NEIN, erfordern das meiste Nachdenken. (Pythagoras)

\title{
C14 Modalpartikel
}

1. Übersicht und Abgrenzung

2. Modalpartikeln

3. Negative Modalpartikeln, Adverbien und Negationspartikel

4. Literatur

\section{1. Übersicht und Abgrenzung ${ }^{1}$}

In diesem Kapitel werden die Modalpartikeln (anscheinend, vielleicht etc.) kurz abgehandelt. Sie werden öfter auch als Modaladverbien bezeichnet und dem Adverb $(\rightarrow \mathrm{C} 4)$ zugeordnet. In Kombination mit einem Responsiv $(\rightarrow \mathrm{C} 10)$ oder allein können sie auf eine Entscheidungsfrage antworten, während Adverbien als Antwort auf eine Ergänzungsfrage dienen können. Anders als Adverbien sind Modalpartikeln nicht phrasenbildend und nur beschänkt koordinierbar. Von der syntaktischen Funktion her gesehen sind sie Satzadverbialia, semantisch betrachtet haben sie weiten Skopus über den ganzen Satz. Die Funktion von Modalpartikeln haben auch einige genuine Adjektive ( $\rightarrow$ C2), die wir - unabhängig von der Homonymiefrage - mitbehandeln. Modale Satzadverbialia können im Mittelfeld des deutschen Satzes die Grenze zwischen Hintergrund und (gewichtetem) Vordergrund markieren (Zifonun/Hoffmann/Strecker 1997: 1538 ff.).

Als Satzadverbialia nehmen die Modalpartikeln eine Sonderstellung ein; sie gestatten es dem Sprecher, sich eben nicht auf $j a$ oder nein festzulegen, sich also das pythagoräische Nachdenken zu ersparen. Damit sind sie einerseits abzugrenzen gegen rein wahrheitsfunktionale Ausdrücke wie z.B. die Negationspartikel nicht, andererseits gegen kontextspezifizierende Ausdrücke wie temporale und lokale Adverbien, z. B. jetzt, dort.

Die Darstellung folgt weitgehend dem Kapitel E 2 3.1.2. in Zifonun/Hoffmann / Strecker 1997. 


\subsection{Semantische Kennzeichnung der Modalpartikeln}

Unter semantischen Gesichtspunkten lassen sich Satzadverbialia durch logische Schlussweisen kennzeichnen und gleichzeitig von den rein wahrheitsfunktionalen Ausdrücken abgrenzen. Offensichtlich gibt es für den Schluss von einem Satz $\mathbf{S}$ mit einem Satzadverbiale auf einen entsprechenden Satz S' ohne das entsprechende Adverbiale drei Möglichkeiten:

\subsubsection{Der Satz $S^{\prime}$ folgt aus dem Satz $S$}

Dies ist der Fall bei den sogenannten assertiven Satzadverbialia, z.B. folgt aus

(1) Es regnet wirklich.

der Satz

(1') Es regnet.

1.2.2. Weder folgt Satz $S^{\prime}$ noch der Satz nicht(S') aus $S$

Dies ist der Fall bei den modal abschwächenden Satzadverbialia, z.B. folgt aus

(2) Es regnet wahrscheinlich.

weder der Satz

(2') Es regnet.

noch der Satz

(2”) Es regnet nicht.

\subsubsection{Der Satz nicht ( $\left.S^{\prime}\right)$ folgt aus dem Satz $S$}

Dies ist der Fall bei den negativen Satzadverbialia, z. B. folgt aus

(3) Es regnet niemals.

der Satz

(3') Es regnet nicht.

In der Folge werden diese drei Gruppen ausführlich dargestellt. 


\section{Modalpartikeln}

\subsection{Assertive Modalpartikeln}

Diese Gruppe gliedert sich in die rein assertiven Modalpartikeln und die bewertend assertiven sowie die evidenzbetonend-assertiven Modalpartikeln.

\subsubsection{Rein assertive Modalpartikeln}

$\mathrm{Zu}$ dieser Gruppe gehören die Modalpartikeln bestimmt, gewiss, das veraltete gewisslich, tatsächlich, vermutlich sowie wirklich. Sätze mit diesen assertiven Modalpartikeln vermitteln keine wahrheitsbezogene Information, die über die der entsprechenden Sätze ohne sie hinausgeht, sind also eigentlich redundant. Damit stellt ihre Verwendung einen Verstoß gegen die Grice'schen Maximen der Relevanz (Max. III: Sag nur Relevantes!) und der Modalität (Untermaxime IV, 3: Vermeide Weitschweifigkeit!) dar. Dadurch wird eine Implikatur ausgelöst, die darin besteht, dass der Wahrheitsanspruch unterstrichen wird. Somit sind sie in der Verwendung pragmatisch gesehen den evidenzbetonenden ähnlich. Der Sprecher kann mit ihrer Hilfe den Wahrheitsanspruch eines Satzes hervorheben. Dies geschieht meistens in Kontexten, wo diese Sätze entweder im Kontrast mit modalisierten Aussagen stehen, wie in (4), wo sie als Korrektur eingesetzt werden, wie in (5) oder als Bestätigung fungieren, wie in (6):

(4) Der Geheimdienstmajor Leroy war vermutlich seit Mai 1965, bestimmt aber seit Oktober über die geplante Entführung informiert.

(5) Diese Krankheit tritt klinisch unter dem Bild einer Leberschrumpfung aus. Tatsächlich handelt es sich aber um entzündliche Verwachsungen des Herzbeutels.

(6) Das ist nicht nur unwahrscheinlich im naiven Sinne, sondern auch mathematisch. Tatsächlich vertritt die Sternenphysik die Meinung, dass es höchst unwahrscheinlich sei, dass es ein Gebilde wie die Erde im Kosmos noch einmal gäbe.

\subsubsection{Bewertend-assertive Modalpartikeln}

Bei den bewertend-assertiven Modalpartikeln kommt zu dem wahrheitsfunktionalen Gehalt der Assertivität noch eine Bewertung des Sachverhalts durch den Sprecher hinzu. Eine positive Bewertung 
durch den Sprecher liegt vor bei den Modalpartikeln begrüßenswerterweise, dankenswerterweise, erfreulicherweise, glücklicherweise, gottseidank, gottlob u. ä.:

(6) Gottlob ist die Operation glücklich verlaufen.

(7) Dankenswerterweise bietet das IDS telefonische Beratung bei sprachlichen Problemen an.

Eine weitere Gruppe bilden immerhin, wenigstens, zumindest. Hier ist die positive Bewertung stark eingeschränkt, wie z.B. in der Äußerung eines Wasserballtrainers:

(8) Wir haben zwar hoch verloren, aber immerhin ist niemand ertrunken.

(9) Gottseidank blieb es bei einem Blechschaden.

Eine negative Bewertung bringen zum Ausdruck leider, bedanerlicherweise, unglücklicherweise:

(10) Bedauerlicherweise ist der Zahn nicht zu retten.

Bewertungen, die nicht einfach auf einer positiv-negativ-Skala liegen, bilden abgeleitete Modalpartikeln auf der Basis Adjektiv+er+weise, wobei das jeweilige Adjektiv die Bewertungsdimension spezifiziert. Hierher gehören z. B. rübrenderweise, bemerkenswerterweise, interessanterweise, verständlicherweise etc.:

(11) Bemerkenswerterweise hat sich die Spielstärke von Schachprogrammen in den letzten Jahren rapide entwickelt.

\subsubsection{Evidenzbetonende assertive Modalpartikeln}

$\mathrm{Zu}$ dieser Gruppe gehören die Modalpartikeln bekanntermaßen, bekanntlich, erwiesenermaßen, nachweislich, sicherlich, zweifellos, zweifelsobne, begreiflicherweise, bezeichnenderweise, natürlicherweise, logischerweise etc., sowie die auch als Adjektive verwendeten nachweislich, offenkundig, offensichtlich, selbstverständlich und fraglos.

Mit ihrer Hilfe kann ein Sprecher die Evidenz seiner Aussage betonen durch Anknüpfung an gemeinsames oder als gemeinsam unterstelltes Wissen, Ziele oder Normensysteme:

(12) Zweifellos liegt eine gewisse Gerechtigkeit darin, dass die vielen guten Burgunder auf den Empfängen der Prominenz mit gewissen Beschwerden bezahlt werden müssen. 
Hier gibt es eine Subgruppe, die als stärkste mögliche Evidenz die Notwendigkeit hervorhebt, bei der als folgendes Schlussprinzip gilt:

Der Satz $\mathbf{S}$ mit dem entsprechenden Element impliziert den Satz notwendig ( $\left.S^{\prime}\right)$.

Hierzu gehören die auch als Adjektive verwendeten notwendig, unumgänglich, unausweichlich, unumstößlich sowie die Modalpartikel notwendigerweise; auch Präpositionalphrasen wie mit Notwendigkeit zeigen diese Funktion.

Bei den Elementen dieser Gruppe spielen verschiedene Redehintergründe ${ }^{2}$ eine Rolle:

„Redehintergründe sind Teil des Text- oder Diskurswissens ... (...) In den Redebeiträgen von Sprechern im Diskurs dienen Redehintergründe als handlungs- und interaktionsleitende Orientierungen des Sprechers. Der Sprecher orientiert sich z.B. an der Gesamtheit des ihm zur Verfügung stehenden Wissens über die anstehenden Redegegenstände (EPISTEMISCHER REDEHINTERGRUND), wobei auch aspeziell an stereotypisches Wissen über den normalen, vorhersehbaren Ablauf einer Handlung, eines Geschehens zu denken ist (STEREOTYPISCHER REDEHINTERGRUND). Oder er orientiert sich an sozialen Normen oder Verpflichtungen im Zusammenhang mit dem anstehenden Sachverhaltsentwurf (NORMATIVER REDEHINTERGRUND) bzw. an möglichen Handlungszielen (TELEOLOGISCHER REDEHINTERGRUND) oder aber an individuellen Einstellungen, Interessen, Neigungen und Wünschen (VOLITIVER REDEHINTERGRUND).“ (Zifonun / Hoffmann/Strecker 1997: 1882)

Bei diesen Modalpartikeln sind es teleologische Systeme, Normen oder Wissen, oft auch Informationen kontextueller Art. So in (13) ein explizit erwähntes Gesetz:

(13) Die Hessische Gemeindeordnung schreibt nicht vor, dass Kleinstädte notwendig einen hauptamtlichen Vertreter des Bürgermeisters wählen.

\subsection{Modal abschwächende Modalpartikeln}

Bei Verwendung der Modalpartikeln dieser Gruppe kann von dem Satz mit dem entsprechenden Element weder auf die Wahrheit noch auf die Falschheit des einfachen Satzes geschlossen werden. Hierher gehören angeblich, anscheinend, eventuell, wabrscheinlich, hoffentlich, möglicherweise, vielleicht, normalerweise etc.

2 Das Konzept des ,Redehintergrunds` geht zurück auf Kratzer 1978. 
Durch ihre Verwendung kann ein Sprecher die Übernahme der Verantwortung für den Wahrheitsgehalt des Restsatzes vermeiden. Hier spielen verschiedene Redehintergründe eine wesentliche Rolle. So wird bei der Verwendung von angeblich, anscheinend auf einen nicht sprecherbezogenen Hintergrund, Äußerungen, Evidenzen Anderer etc. Bezug genommen, wenn sich die Wahrheitsüberprüfung für den Sprecher nicht durchführen lässt (14) oder der Sprecher gar Zweifel an der Wahrheit hat (15):

(14) Anscheinend wurde der Scheffauer schon in der Bronzezeit bestiegen.

(15) Angeblich ist Hans, den ich gestern in der Kneipe getroffen habe, seit drei Wochen krank.

Wenn zu vermuten steht, dass dem Sprecher der Wahrheitsgehalt des Restsatzes zugänglich ist, kann die Verwendung von angeblich sogar per Implikatur dessen Falschheit zum Ausdruck bringen, z. B.:

(16) Angeblich habe ich das Referat aus dem Internet gezogen.

Hoffentlich bezieht sich auf einen - meist sprecherbezogenen - volitiven Redehintergrund, z. B.:

(17) Und dann stehst du im Tor und denkst: Hoffentlich mache ich keinen entscheidenden Fehler.

Bei der Verwendung von normalerweise liegt ein stereotypischer Redehintergrund vor:

(18) Normalerweise müsste es im Dezember kälter sein.

Charakteristisch für möglicherweise, vielleicht, eventuell, wabrscheinlicherweise sind epistemische Redehintergründe, z.B.:

(19) Möglicherweise hat der Täter seine Fingerabdrücke verwischt.

(20) Wahrscheinlich ist das von einem Elementarteilchen erzeugte Lichtquant kleiner als eins. 


\section{Negative Modalpartikeln, Adverbien und Negationspartikel}

Bei Verwendung von Ausdrücken dieser Gruppe kann auf die Falschheit des Restsatzes geschlossen werden. Hierher gehören die Negationspartikeln $(\rightarrow \mathrm{C} 15)$ nicht (nebst verstärkenden Erweiterungen wie gar nicht, überhaupt nicht), keinesfalls, keineswegs, die skalaren Ausdrücke beinabe und fast, sowie die kontextbezogenen Adverbien nie, nirgends, nirgendwo.

Nicht bringt die bloße Negation zum Ausdruck, ist das allgemeinste Element dieser Gruppe:

(21) Nicht für die Schule, sondern für das Leben lernen wir.

Bei den skalaren beinabe, fast wird zusätzlich zur Negation ausgedrückt, dass der bestehende Sachverhalt dem negierten sehr ähnlich ist oder dass das Eintreten des Sachverhalts, den der Restsatz ausdrückt, wahrscheinlich war:

(22) Er war fast erfroren, als man ihn in der Ambulanz einlieferte.

Bei nirgends, nirgendwo kommt zusätzlich zur Negation ein lokaler Kontext ins Spiel, bei nie, niemals ein temporaler:

(23) Im ganzen Allgäu fiel nirgends ein Flöckchen Schnee.

(24) Niemals in den letzten Jahren gab es einen so warmen Dezember.

Übertragene Verwendungen sind möglich:

(25) Das wurde nirgends so deutlich wie beim Streit um den Gesundheitsfond.

\section{Literatur}

Bartsch, R. (1972) Adverbialsemantik. Frankfurt/Main: Athenäum

Kratzer, A. (1978) Semantik der Rede. Königstein: Scriptor

Zifonun, G. (1982) Satzadverbien und mögliche Umstände. Ein Versuch über die propositionale Bedeutung und Sprechaktfunktion von vielleicht und sicher. In: Deutsche Sprache, 33-52

Zifonun, G./Hoffmann, L./Strecker, B. et al. (1997) Grammatik der deutschen Sprache. Berlin/New York: de Gruyter 